\title{
All organic multiferroic magnetoelectric complexes with strong interfacial spin-dipole interaction
}

\author{
Yuying Yang ${ }^{1}$, Zhiyan Chen ${ }^{1}$, Xiangqian $\mathrm{Lu}^{1}$, Xiaotao Hao ${ }^{1,2}$ and Wei Qin $\mathbb{D}^{1 凶}$
}

The organic magnetoelectric complexes are beneficial for the development on flexible magnetoelectric devices in the future. In this work, we fabricated all organic multiferroic ferromagnetic/ferroelectric complexes to study magnetoelectric coupling at room temperature. Under the stimulus of external magnetic field, the localization of charge inside organic ferromagnets will be enhanced to affect spin-dipole interaction at organic multiferroic interfaces, where overall ferroelectric polarization is tuned to present an organic magnetoelectric coupling. Moreover, the magnetoelectric coupling of the organic ferromagnetic/ferroelectric complex is tightly dependent on incident light intensity. Decreasing light intensity, the dominated interfacial interaction will switch from spin-dipole to dipole-dipole interaction, which leads to the magnetoelectric coefficient changing from positive to negative in organic multiferroic magnetoelectric complexes.

npj Flexible Electronics (2021)5:22; https://doi.org/10.1038/s41528-021-00120-0

\section{INTRODUCTION}

Spin-dependent interactions, such as Dzyaloshinskii-Moriya interaction $^{1,2}$, Rashba spin-orbit interaction ${ }^{3}$, exchange interaction ${ }^{4}$, have attracted more attention because of the rich physical phenomena. Understanding of the spin-dependent interaction effectively promotes the development of spintronics. Multiferroic magnetoelectric coupling, the interaction between the spin and the ferroelectric orders ${ }^{5-10}$, provides a good platform for the deep understanding of spin-dependent interfacial interactions ${ }^{11-13}$. For example, a pronounced magnetoelectric coupling is generated through the interaction between $\mathrm{Yb}^{3+}$ spins and $\mathrm{Zn}^{2+}$ dipoles at room temperature ${ }^{5,14}$, where the ferroelectric polarization could be changed through applying external magnetic field. For inorganic multiferroic magnetoelectric materials, single-phase materials $^{15}$ and multi-phase multiferroic complexes ${ }^{16,17}$ are fabricated. For some of them ${ }^{17}$, lattice-matched heteroepitaxy is needed to facilitate magnetoelectric coupling measurement. However, organic multiferroic magnetoelectric measurements cannot be affected by lattice mismatch because of the flexibility, which presents a convenient way to study magnetoelectric coupling. Apart from this, the organic multiferroics have the advantage of flexibility, comparing to rigid inorganic multiferroic materials. Thus, the flexible magnetoelectronic devices can be developed in the area of organic materials.

The organic multiferroic magnetoelectric coupling, representing a kind of physical phenomenon, has been observed in some pure organic complexes. For example, magnetoelectric chargetransfer tetrathiafulvalene- $p$-bromanil, it is a magnetically controllable ferroelectricity material, where the spin-Peierls instability occurs owing to the dimerization in the stacking of the building blocks ${ }^{18}$. As a result, magnetic field can effectively tune the strength of spin-exchange interaction and then change the ferroelectric polarization to present magnetoelectric coupling effect. However, the Curie temperature is only $53 \mathrm{~K}(T \mathrm{c}=53 \mathrm{~K})$. Besides magnetically controllable ferroelectrics of the organic charge-transfer complexes, the phenomenon of electric-dipoledriven magnetism also was observed in organic charge transfer salts, $k$-(BEDT-TTF $)_{2} \mathrm{Cu}\left[\mathrm{N}(\mathrm{CN})_{2}\right] \mathrm{Cl}^{19}$, where the tunability of the spin polarization by the electric field was observed below $T \mathrm{c}=$ $25 \mathrm{~K}$. Furthermore, electric field could effectively tune the ferromagnetic polarization in organic conjugated copolymer P3HT-b-P3HCT ${ }^{20}$. Although the breakthroughs are achieved in organic magnetoelectric couplings, the performance still cannot fulfill the requirement of application.

Recently, based on rapid development in polymeric ferroelectrics ${ }^{21,22}$, small molecular ferroelectric ${ }^{23,24}$ and organic charge transfer ferroelectric with high Curie temperature (above room temperature $)^{25,26}$, organic/inorganic hybrid complexes ${ }^{27-30}$ present pronounced magnetoelectric couplings with a coefficient of $21.46 \mathrm{~V} \mathrm{~cm}^{-1} \mathrm{Oe}^{-131}$. Moreover, with recent study on organic ferromagnets, some of them are of well ferromagnetism at room temperature ${ }^{32,33}$. Thus, we dare to think that the organic ferromagnet/ferroelectric complexes are likely to be prepared for the study of all organic magnetoelectric couplings, which will be beneficial for the development on flexible magnetoelectric devices.

Herein, we fabricated all organic ferromagnetic/ferroelectric complex to study magnetoelectric coupling at room temperature. Under the stimulus of external magnetic field, the spin polarization dependence of localization of charge inside organic ferromagnet will be changed to affect spin-dipole interaction in organic multiferroic complexes, where overall ferromagnetic polarization is tuned to present organic magnetoelectric coupling. The mechanism of organic magnetoelectric coupling is totally different from that of inorganic magnetoelectric materials. Furthermore, the magnetic field dependence of dielectric constant provides further information to understand the mechanism of organic magnetoelectric couplings. Moreover, the organic magnetoelectric coupling is also tightly dependent on external light intensity. Changing the incident light intensity could switch the dominated interfacial interaction from spin-dipole to dipole-dipole interaction in all organic ferromagnetic/ferroelectric complexes. 
(a)

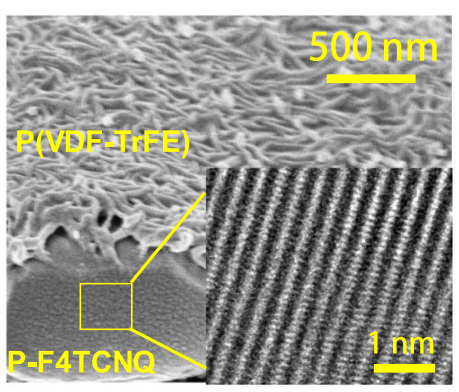

(c)

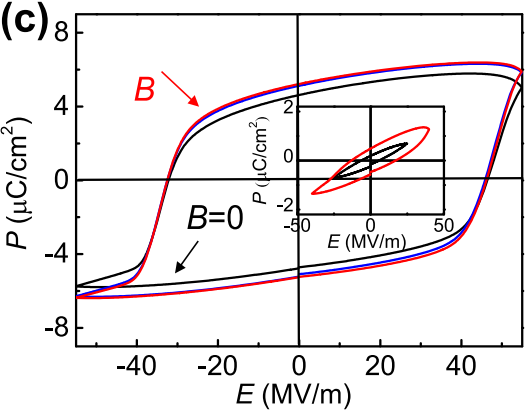

(b)

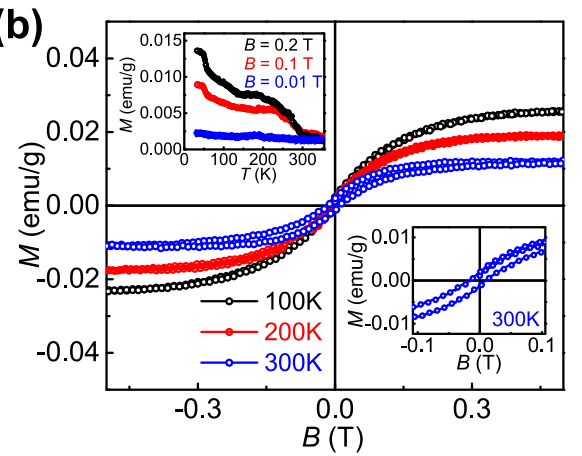

(d)

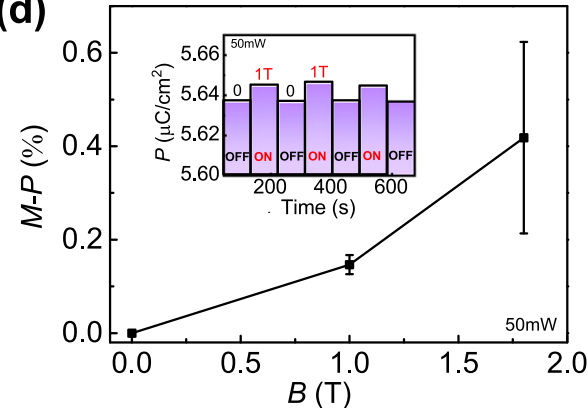

Fig. 1 Ferromagnetic and magnetoelectric coupling. a SEM of the ferromagnetic P-F4TCNQ/ferroelectric P(VDF-TrFE) complexes. The inset shows the HRTEM image of P-F4TCNQ. b M-H loops of ferromagnetic P-F4TCNQ/ferroelectric P(VDF-TrFE) complexes with different temperature. The up inset is the temperature dependence of ferromagnetic magnetization with different applied magnetic fields. $\mathbf{c} P-\mathrm{E}$ loops of ferromagnetic P-F4TCNQ/P(VDF-TrFE) complexes. d Magnetic field-dependent ferroelectric polarization of P-F4TCNQ/P(VDF-TrFE) complexes under light illumination of $50 \mathrm{~mW}$. The ON (OFF) denotes that the external magnetic field is tuned on (off). $M-P=\frac{\mathbf{P}(\mathbf{B})-\mathbf{P}(\mathbf{B}=0)}{\mathbf{P}(\mathbf{B}=0)} \times 100 \%$. The error bars are standard deviations of the three times. ON/OFF cycles measurements with different magnetic fields.

\section{RESULTS AND DISCUSSION}

\section{Characterizations of P-F4TCNQ/P(VDF-TrFE)}

Through adopting the ferroelectric polymer P(VDF-TrFE) combining with the fabricated organic ferromagnet of P-F4TCNQ (pyrene2,3,5,6-tetrafluoro-7,7,8,8-tetracyanoquinodimethane), all organic ferroelectric/ferromagnetic multiferroic magnetoelectric complexes are designed. The high-resolution transmission electron microscopy (HRTEM) of organic charge transfer P-F4TCNQ and scanning electron microscopy (SEM) of multiferroic P(VDF-TrFE)/PF4TCNQ complexes are shown in Fig. 1a. The reason why we choose P-F4TCNQ crystals and P(VDF-TrFE) to study organic magnetoelectric coupling is that P-F4TCNQ is a ferromagnetic material and $\mathrm{P}(\mathrm{VDF}-\mathrm{TrFE})$ is a ferroelectric material. The saturation magnetization of P-F4TCNQ is 10 memu g $^{-1}$ at room temperature. $\mathrm{M}-\mathrm{T}$ (magnetization-temperature) curves (the inset of Fig. $1 \mathrm{~b}$ and Supplementary Fig. 1c) and magnetic force microscopy (Supplementary Fig. $1 \mathrm{~b}$, room temperature) provide further evidences to confirm that P-F4TCNQ is a room temperature ferromagnet. With increasing the temperature, the saturation magnetization decreases. Although the ferromagnetism is very weak for organic ferromagnets, the mechanism of the origin of organic ferromagnetism has been also well studied ${ }^{34-37}$.

\section{Magnetoelectric coupling of P-F4TCNQ/P(VDF-TrFE)}

The P-E (polarization-electric field) loops with the applied voltage of $55 \mathrm{~V}$ are shown in Fig. 1C, where the dipoles are fully polarized to present a well ferroelectric polarization of P-F4TCNQ/P(VDFTrFE) film. However, as shown in the inset of Fig. 1C, when the applied voltages are $25 \mathrm{~V}$ (black line) and $40 \mathrm{~V}$ (red line), respectively, the dipoles cannot be fully polarized. Black line in Fig. $1 \mathrm{C}$ is the P-E loops of multiferroic P-F4TCNQ/P(VDF-TrFE) complexes without the stimulus of external magnetic field. Once applying external magnetic field, both saturation polarization and residual polarization increase (Fig. 1c). We define the dependence of ferroelectric polarization on magnetic field in multiferroic $\mathrm{P}$ F4TCNQ/P(VDF-TrFE) complexes as $\mathrm{M}-\mathrm{P}=\frac{\mathbf{P}(\mathbf{B})-\mathbf{P}(\mathbf{B}=0)}{\mathbf{P}(\mathbf{B}=0)} \times 100 \%$. External magnetic field tunes the magnitude of ferroelectric polarization of multiferroic P-F4TCNQ/P(VDF-TrFE) complexes remarkably. Moreover, a good repeatability of the tunability of ferroelectric polarization by magnetic field is observed (the inset of Fig. 1d). Under the stimulus of magnetic field of $1 \mathrm{~T}$, ferroelectric polarization of the complexes increases from $5.637 \mu \mathrm{C} \mathrm{cm}^{-2}$ to $5.645 \mu \mathrm{C} \mathrm{cm}^{-2}$, which results in a magnetoelectric coefficient of $15.2 \mathrm{~V} \mathrm{~cm}^{-1} \mathrm{Oe}^{-1}$. With further increasing external magnetic field, $\mathrm{M}-\mathrm{P}$ becomes more pronounced (Fig. 1d). However, for the pure ferroelectric $\mathrm{P}(\mathrm{VDF}-\mathrm{TrFE})$ without participation of ferromagnetic PF4TCNQ, electric polarization has no response with the stimulus of external magnetic field (Supplementary Fig. 2).

In the following, we study the mechanism of organic magnetoelectric coupling in multiferroic P-F4TCNQ/P(VDF-TrFE) complexes. Inside the P-F4TCNQ, an electron transferred from donor to acceptor could result in the splitting of up spin and down spin DOS (density of state) (Fig. 2a), which is the main reason for the origin of ferromagnetism in multiferroic P-F4TCNQ/P(VDF-TrFE) complexes. In organic materials, electrons (or holes) prefer to form localized states because of strong electron-lattice coupling ${ }^{38}$. Through changing electron-lattice coupling coefficient, the degree of localization can be tuned to directly affect the magnitude of spin polarization ${ }^{39}$. The magnitude of spin polarization is directly proportional to the charge localization in organic materials. Large spin polarization (SP) in P-F4TCNQ presents a weak ability on the induction of charge density in P(VDF-TrFE) (yellow color) due to the strong charge localization in P-F4TCNQ (Fig. 2b, c). As a result, magnetic field enhanced spin polarization will lead to a more localized state in ferromagnetic P-F4TCNQ, which weakly interacts with the dipole in ferroelectric P(VDF-TrFE) 


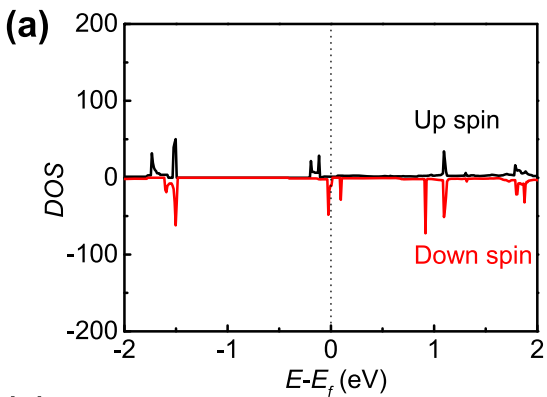

(b)

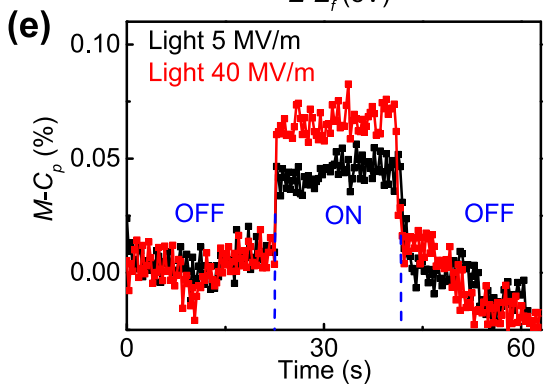

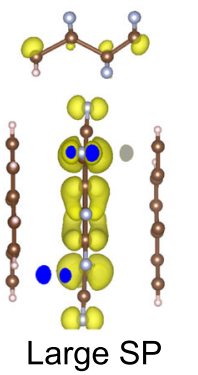

(c)

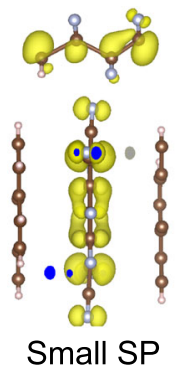

(d)

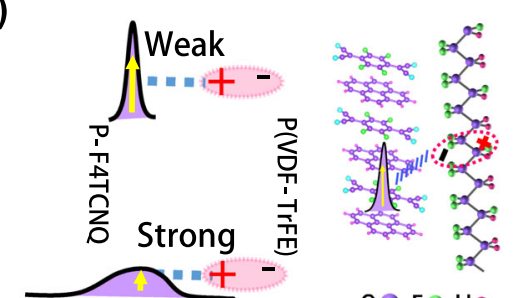

$\mathrm{CO} \mathrm{FOHO}$
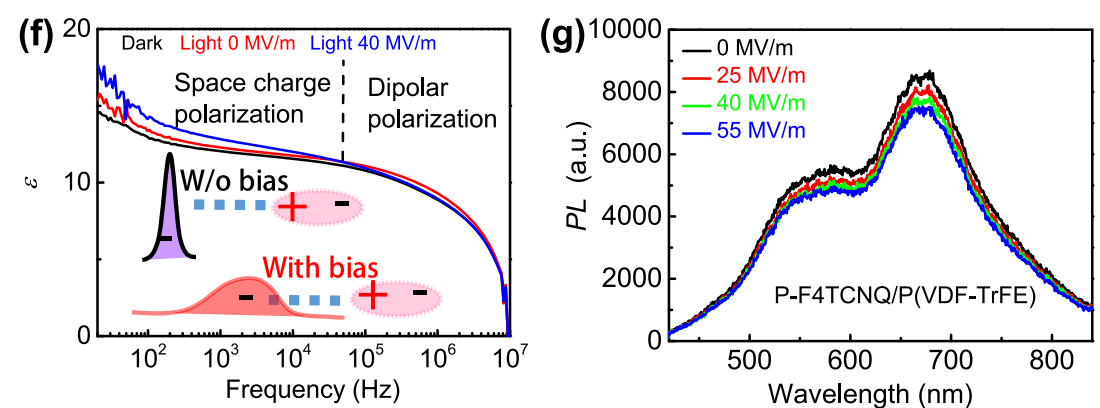

Fig. 2 Theoretical simulation and experiment analysis. a Spin dependence of density of states (DOS) of P-F4TCNQ/P(VDF-TrFE) complexes. Strong $\mathbf{b}$ and weak $\mathbf{c}$ charge interaction at interface of P-F4TCNQ/P(VDF-TrFE). SP denotes spin polarization in FM. With small spin polarization in FM, the interaction between P-F4TCNQ and P(VDF-TrFE) is stronger. $\mathbf{d}$ The diagram of spin-dipole interaction at multiferroic P-F4TCNQ/P (VDF-TrFE) complexes. e Magnetic field dependence of dielectric constant of multiferroic P-F4TCNQ/P(VDF-TrFE) complexes. f Frequency dependence of dielectric constant of multiferroic P-F4TCNQ/P(VDF-TrFE) complexes with/without applying external electric field. $\mathbf{g}$ Electric field dependence of photoluminescence of multiferroic P-F4TCNQ/P(VDF-TrFE) complexes.

(Fig. 2d). In this case, the dipole in P(VDF-TrFE) can be more easily aligned by applied electric field to present larger ferroelectric polarization. Thus, magnetic field could increase the magnitude of ferroelectric polarization (Fig. 1d). Moreover, further increasing the applied magnetic field on F4TCNQ/P(VDF-TrFE) film, the electrons in P-F4TCNQ will become more localized to further weaken the interfacial interaction. As a result, larger magnetic field generates a more pronounced magnitude of M-P for multiferroic complexes (Fig. 1d).

In the case of single ferroelectric P(VDF-TrFE) layer (without interfacial interaction), both the saturation polarization and residual polarization are larger than that of multiferroic $\mathrm{P}$ F4TCNQ/P(VDF-TrFE) complexes with identical thickness (Supplementary Fig. 3). Thus, the interfacial interaction inside the complexes will fix the dipoles in ferroelectric layer to decrease the overall ferroelectric polarization. For the inorganic multiferroic magnetoelectric complexes, such as $\mathrm{BaTiO}_{3}-\mathrm{CoFe}_{2} \mathrm{O}_{4}{ }^{17}, \mathrm{BaTiO}_{3}$ is ferroelectric phase and $\mathrm{CoFe}_{2} \mathrm{O}_{4}$ is ferromagnetic phase. The great elastic interaction between ferroelectric phase and ferromagnetic phase leads to the magnetoelectric coupling effect above room temperature. Thus, the mechanism of organic magnetoelectric coupling is different from that of inorganic magnetoelectric materials.

In addition, it is noted that the error bar of M-P is big when the magnetic field is large (Fig. 1d). Under the stimulus of larger magnetic field, $\sim 2 \mathrm{~T}$, the energy levels of triplet electron-hole pairs will be split, where the crossing between singlet and triplet states will be enhanced in P-F4TCNQ. Because the lifetime of singlet state is much smaller than that of triplet state, singlet and triplet electron-hole pairs (dipoles) in P-F4TCNQ are of different disturbance in interfacial spin-dipole interaction. As a result, during many measurements in magnetic field $(\sim 2 \mathrm{~T})$ dependent on ferroelectric polarization, both singlet and triplet electron-hole pairs have chance to disturb the interfacial interaction. Sometimes the singlet state disturbs interfacial interaction, while sometimes the triplet state disturbs interfacial interaction. This may be the main reason why the error bar of $\mathrm{M}-\mathrm{P}$ is big when the magnetic field is large $(\sim 2 \mathrm{~T})$. Furthermore, magnetic field-dependent dielectric constant of multiferroic P-F4TCNQ/P(VDF-TrFE) complexes provides further evidence that external magnetic field is in favor of the tunability on electric polarization (Fig. 2e). External magnetic field can lead to stronger spin polarization to enhance the localization of electron in ferromagnetic P-F4TCNQ to weaken the interfacial interaction. The dipole in P(VDF-TrFE) can be more easily responded to electric field to enhance dielectric effect.

Besides the tunability of electrons localization by external magnetic field, external electric field could also effectively change the coefficient of electron-lattice coupling to tune the charge localization in P-F4TCNQ. Generally, the lattice vibration-generated phonon contains the acoustic mode and optical mode, and both of them are coupled with electrons (or holes) in organic materials. Once external electric field is applied, the charge splits from the acoustic mode gradually ${ }^{40}$, where the electron-lattice coupling becomes weak. For this case, weaker localized state will be generated to lead to a stronger interfacial interaction (the inset of Fig. 2f). As a result, stronger interfacial interaction will lock the electric polarization. As shown in Fig. $2 \mathrm{f}$, electric dipolar polarization becomes smaller with applying external electric field. Meanwhile, applied external electric field could also increase the dissociation rate of photo-generated electron-hole pairs to enhance the density of space charge in complexes, where the space charge polarization increases (Fig. 2f). In addition, the space charge density determines the performance of luminescence of the complexes. As shown in Fig. 2g, applying external electric field will suppress the recombination of space charge to decrease the strength of photoluminescence (PL).

\section{The tunability of magnetoelectric coupling by changing light intensity}

Based on the above analysis, it is noted that both the charge localization and charge density directly determine the magnetoelectric coupling in organic multiferroic complexes. In organic charge transfer complexes, stronger light illumination could increase the strength of build-in field, where electron-hole pairs become more easily dissociated into space charges ${ }^{41-43}$. Thus, in 


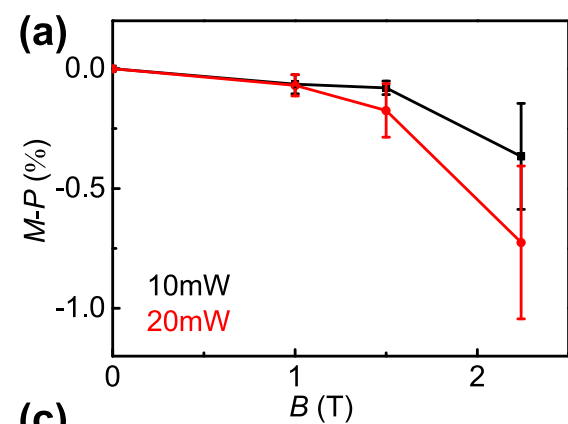

(c)
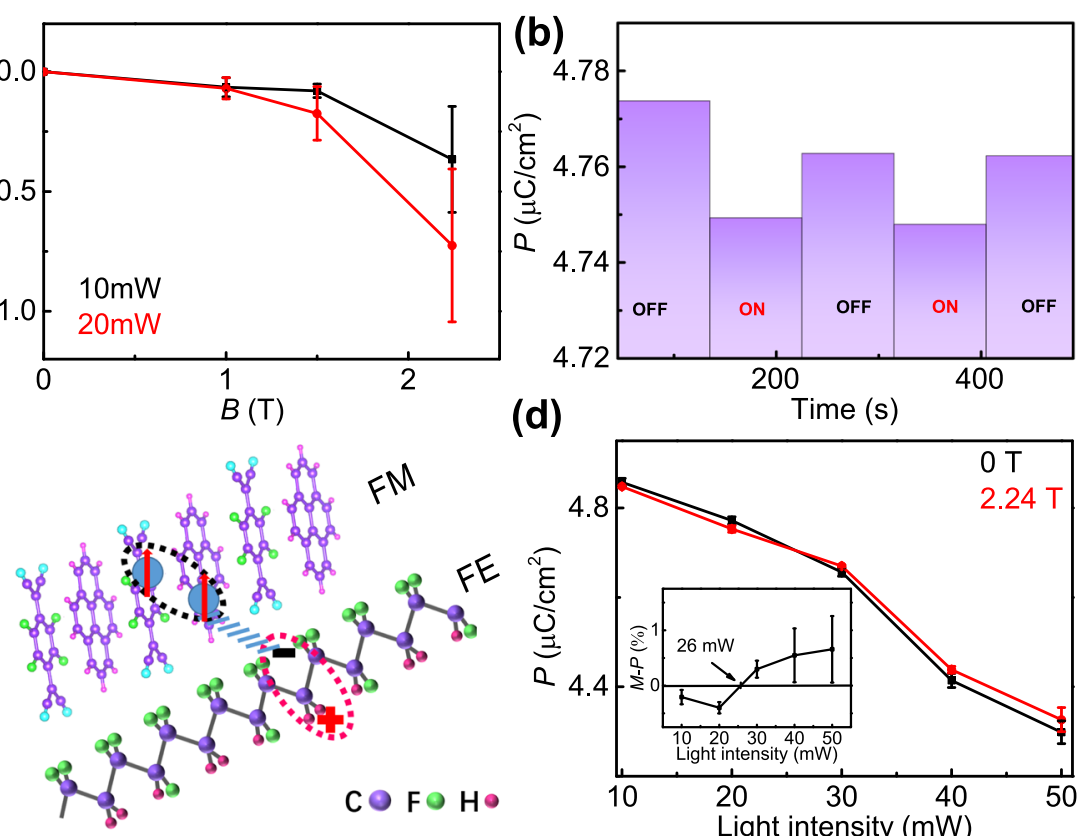

(d)

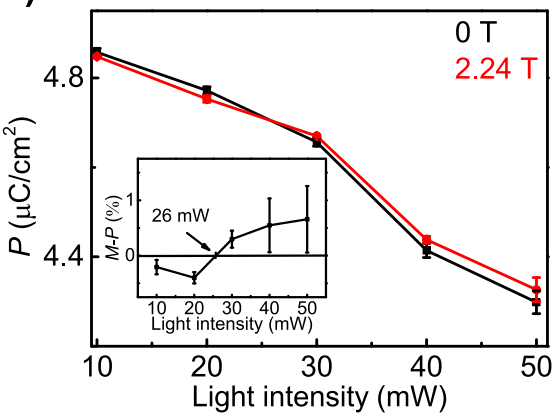

Fig. 3 Magnetoelectric coupling with different light intensities. a Magnetic field dependence of electric polarization of multiferroic P-F4TCNQ/P(VDF-TrFE) complexes under light illuminations of $10 \mathrm{~mW}$ and $20 \mathrm{~mW}$. $\mathbf{b}$ The ON (OFF) in the inset means that magnetic field is tuned on (off). Magnetic field is $2.2 \mathrm{~T}$. c The diagram of dipole-dipole interaction in multiferroic P-F4TCNQ/P(VDF-TrFE) complexes. d Light intensity dependence of electric polarization of multiferroic P-F4TCNQ/P(VDF-TrFE) complexes with and without external magnetic field stimulus. The inset is light intensity dependence on M-P. The error bars are standard deviations of three times. ON/OFF cycles measurements with different magnetic fields.

complexes, the concentration ratio between electron-hole pair and localized electrons (or holes) becomes larger with decreasing light intensity. It means that the dominated interfacial interaction in multiferroic P-F4TCNQ/P(VDF-TrFE) complexes will be switching from spin-dipole interaction to dipole-dipole interaction with decreasing light intensity. Under the light illumination with lower intensity, such as $10 \mathrm{~mW}$, build-in field in organic charge transfer complexes is not large enough to effectively lead to the dissociation of photo-generated electron-hole pairs, and electron-hole pairs in P-F4TCNQ will dominantly interact with dipoles in $\mathrm{P}(\mathrm{VDF}-\mathrm{TrFE})$ to present a dipole-dipole interaction at the complex interface.

Under the light illumination with lower intensity (such as $10 \mathrm{~mW}$, Fig. 3a, b), external magnetic field decreases the electric polarization of the multiferroic P-F4TCNQ/P(VDF-TrFE) complexes, which is completely contrary to the case of that under light illumination of $50 \mathrm{~mW}$ (Fig. 1d). External magnetic field could effectively increase the ratio of triplet electron-hole pairs in organic materials ${ }^{44-46}$. Because of the extremely long lifetime of triplet electron-hole pairs, they will adequately interact with the dipole in $\mathrm{P}(\mathrm{VDF}-\mathrm{TrFE})$ to enhance the interfacial interaction of multiferroic P-F4TCNQ/P(VDF-TrFE) complexes (the diagram of Fig. 3c). Thus, if the dipole-dipole interaction is dominated at the interface, external magnetic field will decrease electric polarization of multiferroic P-F4TCNQ/P(VDF-TrFE) complexes (Fig. 3a). It should be noted that the threshold light intensity is $26 \mathrm{~mW}$, where the interactions of the spin-dipole and dipole-dipole will reach a balance under the stimulus of external magnetic field. Thus, magnetic field cannot tune the electric polarization of multiferroic P-F4TCNQ/P(VDF-TrFE) complexes when the incident light intensity is $26 \mathrm{~mW}$. When the light intensity increases from $10 \mathrm{~mW}$ to $20 \mathrm{~mW}$, the density of photo-generated electron-hole pairs in P-F4TCNQ will increase, where the dipole-dipole interaction at the interface of the multiferroic complexes becomes more pronounced. As a result, stronger interfacial interaction will fix the dipoles in P(VDF-TrFE) to lead to a reduction of overall polarization in multiferroic P-F4TCNQ/P(VDF-TrFE) complexes (Fig. 3d). Furthermore, for the case of larger density of electron-hole under $20 \mathrm{~mW}$ light illumination, external magnetic field can effectively increase the ratio of triplet electron-hole pairs to further enhance the interfacial interaction due to the longer lifetime of triplet electron-hole pairs. As a result, magnetic field dependence of ferroelectric polarization in multiferroic P-F4TCNQ/ $\mathrm{P}(\mathrm{VDF}-\mathrm{TrFE})$ complexes is more pronounced under the stimulus of $20 \mathrm{~mW}$, comparing to that of $10 \mathrm{~mW}$ (the inset of Fig. 3d).

In summary, we have fabricated all organic ferromagnetic PF4TCNQ/ferroelectric P(VDF-TrFE) complexes to realize magnetoelectric coupling at room temperature. Under the stimulus of external magnetic field, the spin polarization in organic ferromagnets is enhanced to generated localized state of the electron, where overall ferroelectric polarization is increased to present a magnetoelectric coupling coefficient of $15.2 \mathrm{~V} \mathrm{~cm}^{-1}$ $\mathrm{Oe}^{-1}$. Ferroelectric/ferromagnetic interfacial coupling is determined by both spin-dipole interaction and dipole-dipole interaction. If the incident light intensity is smaller than $26 \mathrm{~mW}$, the dominated interfacial interaction at the multiferroic P-F4TCNQ/P(VDF-TrFE) complexes will switch from the spin-dipole interaction to dipole-dipole interaction, where magnetic field decreases the electric polarization of multiferroic P-F4TCNQ/P(VDF-TrFE) complexes. Moreover, it should be noted that one of the key challenges for the improvement in magnetoelectric coupling is how to improve the performance of organic ferromagnetic materials. Recently, many organic radical materials have been fabricated with good luminescence and stability ${ }^{47,48}$, and "spin density tuning" strategy is proposed to obtain a triple ground-state-conjugated diradical material with great stability ${ }^{49}$. Thus, it is expected that crystals based on radical materials could improve the performance of organic ferromagnetism. Overall, all organic multiferroic magnetoelectric complexes will promote the development of flexible magnetoelectric devices. 


\section{METHODS}

\section{Preparation of samples}

Pyrene, P(VDF-TrFE) were purchased from SIGMA-ALDRICH. 2,3,5,6-tetrafluoro-7,7,8,8-tetracyanoquinodimethane (F4TCNQ) was purchased from $3 \mathrm{~A}$ Chemical Company. They were used without further treatment. Pyrene and F4TCNQ were dissolved with 1:1 molar ratio in chloroform $(1-3 \mathrm{mg}$ $\mathrm{mL}^{-1}$ ). Dissolve $\mathrm{P}(\mathrm{VDF}-\mathrm{TrFE})$ in tetrahydrofuran (THF) at a concentration of 30 and $50 \mathrm{mg} \mathrm{mL}^{-1}$. Spin-coat P(VDF-TrFE) (mg mL $\left.\mathrm{mb}^{-1}\right)$, drip P-F4TCNQ solution, and spin-coat P(VDF-TrFE) ( $\mathrm{mg} \mathrm{mL}^{-1}$ ) on the ITO in turn, and deposit aluminum $(150 \mathrm{~nm})$

\section{Structural characterization}

We performed the TEM measurements by a Cs-corrected FEI Titan transmission electron microscope at $300 \mathrm{kV}$. We got the HRTEM image by a Gatan K2 camera using direct detection. To decrease the damages from beam, the parameters of dose rate and total electron dose were $0.5 \mathrm{e}$ $\mathrm{A}^{-2} \mathrm{~S}^{-1}$ and 5 e $\mathrm{A}^{-2}$ respectively. Nova Nano SEM 450 (FEI) instrument was used to characterize the SEM of multiferroic P-F4TCNQ/P(VDF-TrFE) complexes.

\section{Magnetoelectric coupling characterization}

P-F4TCNQ crystals on the silicon wafers were put on the measurement stage of MFM. The MFM image was measured through Dimension Icon (Veeco Instruments Inc.). The MFM probe with coating $\mathrm{Co} / \mathrm{Cr}$ was used. We first scanned the topography of the P-F4TCNQ crystal, then lifted the MFM probe to record the MFM image. M-H loops were performed by SQUID and VSM (Lakeshore 8604). Radiant Precision LC was used to characterize P-E loops. The photoluminescence was accomplished by an FLS980 spectrometer. The dielectric-constant-dependent properties were carried out with impedance analyzer (Keysight, E4990A). The ab initio calculations described here were implemented in the Quantum ESPRESSO program with norm-conserving pseudo potentials. The electronic band structures were calculated using the Heyd-Scuseria-Ernzerhof (HSE) exchange-correlation functional.

\section{DATA AVAILABILITY}

The data that support the findings of this study are available from the authors on reasonable request, see author contributions for specific data sets.

Received: 31 May 2021; Accepted: 5 August 2021; Published online: 19 August 2021

\section{REFERENCES}

1. Lin, W. et al. Perpendicular magnetic anisotropy and Dzyaloshinskii-Moriya interaction at an oxide/ferromagnetic metal interface. Phys. Rev. Lett. 124, 217202 (2020).

2. Arora, M., Shaw, J. M. \& Nembach, H. T. Variation of sign and magnitude of the Dzyaloshinskii-Moriya interaction of a ferromagnet with an oxide interface. Phys. Rev. B 101, 054421 (2020).

3. Lin, W. et al. Interface-based tuning of Rashba spin-orbit interaction in asymmetric oxide heterostructures with 3d electrons. Nat. Commun. 10, 3052 (2019).

4. Liao, Y.-C. et al. Understanding the switching mechanisms of the antiferromagnet/ferromagnet heterojunction. Nano Lett. 20, 7919-7926 (2020).

5. Long, J. et al. Room temperature magnetoelectric coupling in a molecular ferroelectric ytterbium(III) complex. Science 367, 671-676 (2020).

6. Wang, $\mathrm{H}$. et al. Large polarization switching and high-temperature magnetoelectric coupling in multiferroic $\mathrm{GaFeO}_{3}$ systems. Inorg. Chem. 60, 225-230 (2021).

7. Fina, I. et al. The direct magnetoelectric effect in ferroelectric-ferromagnetic epitaxial heterostructures. Nanoscale 5, 8037-8044 (2013).

8. Chen, A. et al. Giant nonvolatile manipulation of magnetoresistance in magnetic tunnel junctions by electric fields via magnetoelectric coupling. Nat. Commun. 10, 243 (2019).

9. $\mathrm{Yi}$, D. et al. Tailoring magnetoelectric coupling in $\mathrm{BiFeO}_{3} / \mathrm{La}_{0.7} \mathrm{Sr}_{0.3} \mathrm{MnO}_{3}$ heterostructure through the interface engineering. Adv. Mater. 31, 1806335 (2019).

10. Pesquera, D. et al. Large magnetoelectric coupling in multiferroic oxide heterostructures assembled via epitaxial lift-off. Nat. Commun. 11, 3190 (2020).

11. Eerenstein, W., Mathur, N. D. \& Scott, J. F. Multiferroic and magnetoelectric materials. Nature 442, 759-765 (2006).

12. Ma, J., Hu, J., Li, Z. \& Nan, C. W. Recent progress in multiferroic magnetoelectric composites: from bulk to thin films. Adv. Mater. 23, 1062-1087 (2011).
13. Vaz, C. A. F., Hoffman, J., Ahn, C. H. \& Ramesh, R. Magnetoelectric coupling effects in multiferroic complex oxide composite structures. Adv. Mater. 22, 2900-2918 (2010).

14. Zhou, Y. \& Han, S.-T. Room-temperature magnetoelastic coupling. Science 367, 627-628 (2020)

15. Wang, J. et al. Epitaxial $\mathrm{BiFeO}_{3}$ multiferroic thin film heterostructures. Science 299 1719-1722 (2003).

16. Choi, H. K. et al. Hierarchical templating of a $\mathrm{BiFeO}_{3}-\mathrm{CoFe}_{2} \mathrm{O}_{4}$ multiferroic nanocomposite by a triblock terpolymer film. ACS Nano 8, 9248-9254 (2014).

17. Zheng, $\mathrm{H}$. et al. Multiferroic $\mathrm{BaTiO}_{3}-\mathrm{CoFe}_{2} \mathrm{O}_{4}$ nanostructures. Science 303 661-663 (2004).

18. Kagawa, F., Horiuchi, S., Tokunaga, M., Fujioka, J. \& Tokura, Y. Ferroelectricity in a one-dimensional organic quantum magnet. Nat. Phys. 6, 169 (2010).

19. Lunkenheimer, P. et al. Multiferroicity in an organic charge-transfer salt that is suggestive of electric-dipole-driven magnetism. Nat. Mater. 11, 755-758 (2012).

20. Lohrman, J. et al. All conjugated copolymer excitonic multiferroics. Adv. Mater. 25, 783-787 (2013)

21. Lu, Y. Y., Claude, J., Neese, B., Zhang, Q. M. \& Wang, Q. A modular approach to ferroelectric polymers with chemically tunable Curie temperatures and dielectric constants. J. Am. Chem. Soc. 128, 8120-8121 (2006).

22. Hafner, J. et al. Multi-scale characterisation of a ferroelectric polymer reveals the emergence of a morphological phase transition driven by temperature. Nat. Commun. 12, 152 (2021)

23. Fu, D. W. et al. Diisopropylammonium bromide is a high-temperature molecular ferroelectric crystal. Science 339, 425-428 (2013).

24. Fu, D. W. et al. Diisopropylammonium chloride: a ferroelectric organic salt with a high phase transition temperature and practical utilization level of spontaneous polarization. Adv. Mater. 23, 5658-5662 (2011).

25. Tayi, A. S. et al. Room-temperature ferroelectricity in supramolecular networks of charge-transfer complexes. Nature 488, 485-489 (2012).

26. Qin, W. et al. External stimuli controlled multiferroic charge-transfer crystals. Nano Res. 9, 925-932 (2016).

27. Zhou, L. et al. Self-assembled core-shell $\mathrm{CoFe}_{2} \mathrm{O}_{4} @ \mathrm{BaTiO}_{3}$ particles loaded P(VDFHFP) flexible films with excellent magneto-electric effects. Appl. Phys. Lett. 111, 032903 (2017)

28. Mori, K. \& Wuttig, M. Magnetoelectric coupling in terfenol-D/polyvinylidenedifluoride composites. Appl. Phys. Lett. 81, 100-101 (2002).

29. Nan, C. W., Li, M., Feng, X. Q. \& Yu, S. W. Possible giant magnetoelectric effect of ferromagnetic rare-earth-iron-alloys-filled ferroelectric polymers. Appl. Phys. Lett 78, 2527-2529 (2001).

30. Zhai, J. Y., Dong, S. X., Xing, Z. P., Li, J. F. \& Viehland, D. Giant magnetoelectric effect in Metglas/polyvinylidene-fluoride laminates. Appl. Phys. Lett. 89, 083507 (2006).

31. Fang, Z. et al. Enhancing the magnetoelectric response of Metglas/polyvinylidene fluoride laminates by exploiting the flux concentration effect. Appl. Phys. Lett. 95, 112903 (2009)

32. Wei, M., Fan, Y. \& Qin, W. Progress of organic magnetic materials. Sci. China-Phys. Mech. Astron. 62, 977501 (2019).

33. Wang, Z. \& Qin, W. Organic magnetoelectric and optomagnetic couplings: perspectives for organic spin optoelectronics. NPG Asia Mater. 13, 17 (2021).

34. Qin, W. et al. Room temperature multiferroicity of charge transfer crystals. ACS Nano 9, 9373-9379 (2015).

35. Yang, Y. Y. et al. Anisotropic magnetoelectric coupling and Cotton-Mouton effects in the organic magnetic charge-transfer complex pyrene- $\mathrm{F}_{4}$ TCNQ. ACS Appl. Mater. Interfaces 10, 44654-44659 (2018).

36. Wang, Z. X. et al. Organic chiral charge transfer magnets. ACS Nano 13, 4705-4711 (2019).

37. Wei, M. M. et al. Organic multiferroic magnetoelastic complexes. Adv. Mater. 32, 6 (2020)

38. Waragai, K., Akimichi, H., Hotta, S., Kano, H. \& Sakaki, H. Charge-transport in thinfilms of semiconducting oligothiophenes. Phys. Rev. B 52, 1786-1792 (1995).

39. Hou, D., Qiu, J., Xie, S. \& Saxena, A. Charge-induced spin polarization in thiophene oligomers. N. J. Phys. 15, 073044 (2013).

40. Liu, X. et al. Effect of the electric field mode on the dynamic process of a polaron. Phys. Rev. B 74, 172301 (2006).

41. Zhang, X., Wei, M. M. \& Qin, W. Magneto-open-circuit voltage in organic-inorganic halide perovskite solar cells. Appl. Phys. Lett. 114, 4 (2019).

42. Xu, X., Xu, P., Hao, Y. \& Qin, W. Exploring the effects of optically generated dipoles on organic photodetector infrared detection. Org. Electron. 45, 222-226 (2017).

43. Gelinas, S. et al. Ultrafast long-range charge separation in organic semiconductor photovoltaic diodes. Science 343, 512-516 (2014).

44. Qin, W. et al. Multiferroicity of carbon-based charge-transfer magnets. Adv. Mater 27, 734-739 (2015).

45. Qin, W., Jasion, D., Chen, X. M., Wuttig, M. \& Ren, S. Q. Charge-transfer magnetoelectrics of polymeric multiferroics. ACS Nano 8, 3671-3677 (2014). 
46. Chen, Z. Y. \& Qin, W. Spin properties and electronic structure in organic ternary crystals. J. Phys. Chem. C. 124, 15634-15638 (2020).

47. $\mathrm{Ai}, \mathrm{X}$. et al. Efficient radical-based light-emitting diodes with doublet emission. Nature 563, 536-540 (2018)

48. Xue, Y., Guo, P., Yip, H.-L., Li, Y. \& Cao, Y. General design of self-doped small molecules as efficient hole extraction materials for polymer solar cells. J. Mater. Chem. A 5, 3780-3785 (2017).

49. Wang, Z.-Y. et al. A stable triplet-ground-state conjugated diradical based on a diindenopyrazine skeleton. Angew. Chem., Int. Ed. 60, 4594-4598 (2021).

\section{ACKNOWLEDGEMENTS}

This work was supported by the NSFC (Grant Nos. 11774203 and 91963103), Taishan Scholar Foundation of Shandong Province (tsqn201812007), Shandong Province Outstanding Youth Foundation (ZR2020JQ02), Major Program of Shandong Province Natural Science Foundation (ZR2019ZD43), Shandong University Multidisciplinary Research and Innovation Team of Young Scholars (2020QNQT013).

\section{AUTHOR CONTRIBUTIONS}

$Y$. Y prepared crystals and did the measurement on the magnetic field-dependent P-E loops. Z. C. analyzed the data, X. L. did the theoretical simulation. Y. Y. and W. Q. wrote the manuscript, $X$. T. revised the manuscript, and $W$. $Q$. guided the project.

\section{COMPETING INTERESTS}

The authors declare no competing interests.

\section{ADDITIONAL INFORMATION}

Supplementary information The online version contains supplementary material available at https://doi.org/10.1038/s41528-021-00120-0.

Correspondence and requests for materials should be addressed to W.Q.

Reprints and permission information is available at http://www.nature.com/ reprints

Publisher's note Springer Nature remains neutral with regard to jurisdictional claims in published maps and institutional affiliations.

\section{(c) (i)}

Open Access This article is licensed under a Creative Commons Attribution 4.0 International License, which permits use, sharing, adaptation, distribution and reproduction in any medium or format, as long as you give appropriate credit to the original author(s) and the source, provide a link to the Creative Commons license, and indicate if changes were made. The images or other third party material in this article are included in the article's Creative Commons license, unless indicated otherwise in a credit line to the material. If material is not included in the article's Creative Commons license and your intended use is not permitted by statutory regulation or exceeds the permitted use, you will need to obtain permission directly from the copyright holder. To view a copy of this license, visit http://creativecommons. org/licenses/by/4.0/.

(c) The Author(s) 2021 